\title{
A Gigabit VLC receiver that incorporates a fluorescent antenna and a SiPM.
}

\author{
Wajahat Ali, Pavlos P. Manousiadis, Dominic C O’Brien, Graham A Turnbull, Ifor D. W. Samuel and \\ Steve Collins
}

\begin{abstract}
In this paper we demonstrate a novel optical receiver for visible light communications that combines a fluorescent antenna and a silicon photomultiplier (SiPM). The fluorescent antenna is configured in a slab geometry to collect and absorb an incoming optical data signal, and to waveguide the resulting fluorescence to an edge where it is detected by the SiPM. The antenna incorporates the fluorophore, pentafluorene, selected for its very high bandwidth of $245 \mathrm{MHz}$, high photoluminescence quantum yield of $90 \%$, and emission spectrum that matches the wavelengths most efficiently detected by the SiPM. The performance of a receiver, comprising the fluorescent antenna and a J-series 30020 SiPM manufactured by ON Semiconductor, was assessed in a $30 \mathrm{~cm}$ data link with a $405 \mathrm{~nm}$ GaN laser diode transmitter, both in the dark and under 500 lux of ambient white light. The fluorescent antenna successfully rejects ambient light by a factor of 20, limited by extrinsic scattering in the thin film. Using on-off-keying with decision feedback equalization, a maximum data rate of $1.4 \mathrm{Gbps}$ was demonstrated in 500 lux of ambient light. Using the pentafluorene antenna plus a BG3 filter, provides a 200 fold suppression of ambient light, and can increase the data rate of the receiver at low signal powers by up to a factor of 2 . When operating in ambient light the composite receiver requires only 2.5 times more signal power than when it operates in the dark, with this power penalty reducing to a factor of 1.2 at $1 \mathrm{Gbps}$.
\end{abstract}

Index Terms-Fluorescence, fluorescent concentrator, Silicon photomultiplier (SiPM), single-photon avalanche photodiode (SPAD), Optical receivers, visible light communications (VLC), optical wireless communications (OWC), Li-Fi, fluorene, organic semiconductor

\section{INTRODUCTION}

$\mathrm{T}$ HE field of visible light communications (VLC) is driving forward new approaches for wireless data transmission, and accurate indoor positioning and tracking [1,2]. The ability to use VLC to transmit data will be particularly important in situations, such as some factories, where RF interference prevents RF data transmission. However, the most widespread use of VLC is likely to be to complement, or even replace, WiFi.

Submitted on $1^{\text {st }}$ March 2021. This work was supported by EPSRC grants EP/R00689X/1 and EP/R005281/1.

Wajahat Ali and Pavlos Manousiadis contributed equally to this paper.

Wajahat Ali, Dominic O'Brien and Steve Collins are with Department of Engineering Science, University of Oxford, Oxford OX1 3PJ, UK. (emails wajahat.ali@eng.ox.ac.uk,

steve.collins@eng.ox.ac.uk)
The capacity of the VLC channel depends upon a combination of channel bandwidth and the signal to noise ratio (SNR) at the receiver output. The bandwidth of many VLC systems is low compared with the desired data rate but they have a high SNR. In these circumstances, Orthogonal Frequency Division Multiplexing (OFDM) has been used to obtain high-data rates[3-5]. For example, 15.73 Gbps has been transmitted over $1.6 \mathrm{~m}$ using OFDM, wavelength division multiplexing and LEDs with four different colours [3]. When OFDM and WDM were combined with laser diode transmitters and beam steered $35 \mathrm{Gbps}$ can be transmitted over $4 \mathrm{~m}$ [4]. Alternatively, laser diodes and polarization multiplexing has been used to transmit 40.665 Gbps over $2 \mathrm{~m}$ [5]. These VLC systems [3-5] provided high-speed connectivity by focusing on WDM and transmitter design however, their receivers offered a limited FOV. A major challenge for VLC is to develop optical receivers that can combine high sensitivity with high bandwidth and a wide field-of-view, whilst rejecting ambient light.

One recent advance to maximize a VLC receiver's SNR is to replace the photodiode with a photon counting silicon photomultiplier (SiPM). These devices are very sensitive. However, ambient light generates additional noise at the output of the SiPM and can also saturate its response[6]. Since ambient light includes photons with wavelengths from $400 \mathrm{~nm}$ to $700 \mathrm{~nm}$ optical filters improve the performance of VLC receivers. In particular, the best results with a SiPM receiver have been obtained using a multi-layer dielectric optical filter with a $10 \mathrm{~nm}$ passband in front of the SiPM [7]. Unfortunately, this significantly reduces the field-of-view of the receiver.

A conventional approach to reducing the impact of ambient light on a SiPM, without reducing its FoV, would be to use absorption filters to block unwanted wavelengths. An alternative approach is to use a fluorophore that preferentially absorbs the transmitter's wavelength within an optical element that can also concentrate light. Fluorescent concentrators are optical elements that absorb light from the transmitter over an extended area and concentrate the re-emitted fluorescence onto a small photodiode [8,9]. Since fluorescence does not conserve

Pavlos Manousiadis, Graham Turnbull and Ifor Samuel are with the Organic Semiconductor Centre, SUPA, School of Physics and Astronomy, University of St Andrews, St Andrews KY16 9SS, UK (emails pm84@ st-andrews.ac.uk, gat@st-andrews.ac.uk andidws@st-andrews.ac.uk.

The research material supporting this publication can be accessed by contacting steve.collins@eng.ox.ac.uk. 
TABLE.1: Key Parameters of 30020 [11]

\begin{tabular}{|c|c|}
\hline Parameter & \\
\hline Number of Microcells & 14410 \\
\hline Microcells active area diameter $((\mu \mathrm{m}))$ & 20 \\
\hline Fill factor $(\%)$ & 62 \\
\hline Recovery Time $(\mathrm{ns})$ & $15 \mathrm{~ns}$ \\
\hline Photon Detection Efficiency $(405 \mathrm{~nm})$ & $0.38(@ 5 \mathrm{~V})$ \\
\hline Dark Count Rate $(\mathrm{MHz})$ & $1.2($ @ 5V) \\
\hline Pulse width $(\mathrm{ns})$ & 1.4 \\
\hline
\end{tabular}

etendue this approach can simultaneously achieve a high optical gain and a wide field-of-view [10].

The wavelength-selective absorption in fluorescent optical elements means that they can also function as a wide field-ofview optical filter. Combining these optical elements with SiPMs would potentially create a new, very sensitive receiver that can operate in ambient light and have a wide FoV. For this scenario, the most important properties of the fluorescent component are the selective response to the transmitted wavelength and their wide field of view, and it is more appropriate to regard them as selective fluorescent antennas rather than concentrators.

In this paper we present the first example of a VLC receiver formed from the combination of a fluorescent antenna with a SiPM. The fluorescent antenna incorporates the fluorophore, pentafluorene $(5 \mathrm{~F})$, selected for its significantly higher bandwidth that previously used fluorophores, a photoluminescence quantum yield (PLQY) of 90\%, and light emission in the range of wavelengths most efficiently detected by the chosen SiPM. Results are presented that show that the selective response of the $5 \mathrm{~F}$ antenna to light from the transmitter improves the performance of the SiPM receiver.

The paper is organized as follows. Section II contains a description of the chosen SiPMs, whilst section III describes the selection of a fluorophore to work together with the SiPM and its characterization. The results of a rectangular antenna that incorporates a thin layer containing the selected fluorophore $(5 \mathrm{~F})$ are presented in section IV. Section $\mathrm{V}$ then contains the results of experiments in which OOK data was transmitted to a receiver formed by the $\mathrm{SiPM}$ and the $5 \mathrm{~F}$ antenna. Finally, concluding remarks are included in section VI.

\section{CHARACTERISTICS OF THE SELECTED SiPM}

A SiPM is an array of microcells, each of which contains an avalanche photodiode (APD) in series with a quenching device. The photon counting capability of a SiPM is then achieved by biasing the APD above its breakdown voltage. When a photon creates an electron-hole pair it then initiates an avalanche of electron-hole pairs. The presence of the quenching device means that this avalanche discharges the capacitance of the APD. In addition to quenching the avalanche the resulting sudden change in voltage creates a pulse that can be detected on an output shared by all the microcells. The duration of the SiPM's output pulses will cause inter-symbol interference (ISI) for data rates with symbol times shorter than the output pulses. Consequently, the duration of the output pulses is an important parameter when selecting a SiPM for use in a VLC receiver. The $\mathrm{J}$ series of SiPMs manufactured by ON Semiconductor

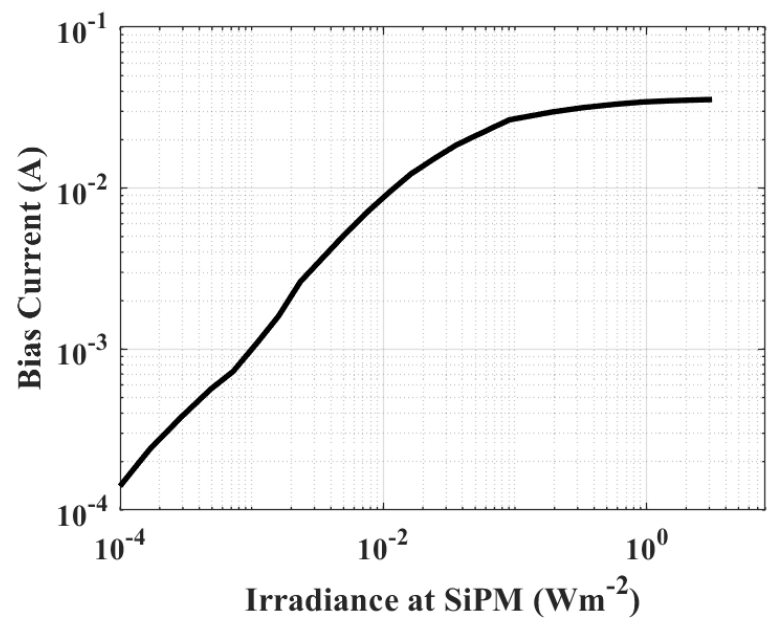

Fig. 1 The relationship between the irradiance falling on a 30020 SiPM and the current required to sustain the overvoltage.

have fast output pulses and therefore a J series 30020, whose characteristics are listed in table 1 , has been used in VLC receivers.

The fast output pulses of the $\mathrm{J}$ series SiPMs arise from the sudden discharge of a microcell followed by a slower recovery process. Consequently, these pulses have a rapid rising edge followed by an exponential decay. In the case of a J Series 30020 SiPM the full-width at half maximum of these pulses is $1.4 \mathrm{~ns}$, which corresponds to a time constant of an exponential decay of $0.64 \mathrm{~ns}$. The relationship between the time constant of the exponential decay, $\tau$, and the corresponding $3 \mathrm{~dB}$ bandwidth

$$
f_{3 d B}=1 /(2 \pi \tau)
$$

means that the bandwidth of the fast output pulses of this SiPM is $249 \mathrm{MHz}$.

Once the avalanche process is quenched the microcell has to recharge. However, during this recharge or recovery time the microcell's ability to detect photons is reduced. The impact of the recovery time on the response of the SiPM has been determined by exposing the SiPM to different irradiances from a $405 \mathrm{~nm}$ laser diode. The response of the SiPM was then characterized by measuring the current required to maintain the SiPM bias voltage. Since this current is required to recharge the microcells it is proportional to the rate at which they are discharged. At low irradiances the time between photons arriving at each microcell is longer than the recovery time and the rate at which microcells discharge, and hence the measured current, are proportional to the irradiance. However, as the irradiance increases the proportion of photons arriving during a recovery time, which are harder to detect, increases. The result is a non-linear relationship between the irradiance on the SiPM and the rate at which microcells discharge. This creates the nonlinear relationship between the irradiance on the SiPM and the current supplied by the bias voltage source shown in Fig. 1.

Ambient light rejection is a challenge for all VLC receivers, but their non-linear response and high sensitivity makes this a particular challenge for SiPMs. For example, previous results showed that ambient light reduces the sensitivity of a SiPM receiver by a factor of 60[6]. Narrowband interference filters have previously been used to protect the SiPM from ambient light. However, the resulting restriction of the receiver's field 

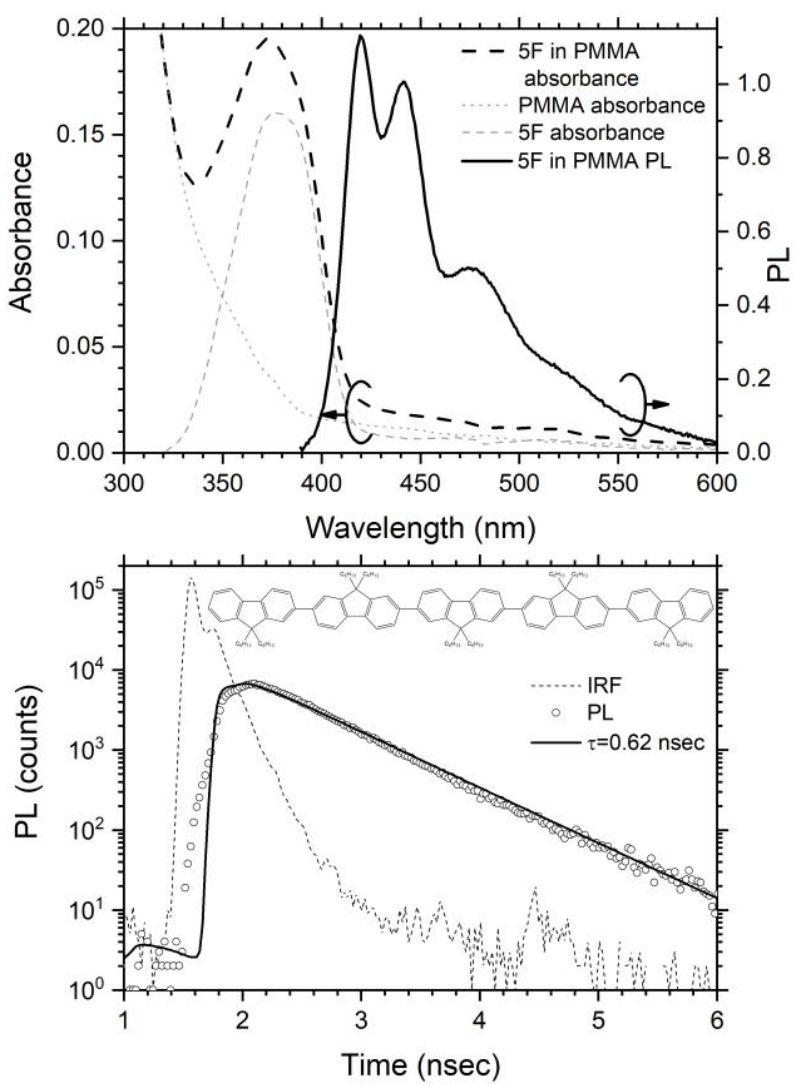

Fig 2. a) Absorption and emission (excited at $379 \mathrm{~nm}$ ) spectra of the $5 \mathrm{~F}$ in PMMA films on glass. Also shown is the absorption of a neat film of PMMA on glass, and of 5F on fused silica. b) The chemical structure of pentafluorene $(5 \mathrm{~F})$ and PL versus time measurement, following excitation at $379 \mathrm{~nm}$ and detection at $450-630 \mathrm{~nm}$.

of view means that they are sensitive to changes of alignment. The resulting problems can be avoided by using a SiPM with a fluorescent antenna rather than an interference filter.

\section{FLUOROPHORE SELECTION AND CHARACTERIZATION}

The bandwidth of a receiver is one of its most important characteristics. A feature of fluorescence not shared by optical filters is that the lifetime of the fluorophore's excited state means that fluorescent optical elements have a finite bandwidth. In order to avoid reducing the bandwidth of a receiver the bandwidth of the fluorophore in a fluorescent antenna should ideally be larger than the bandwidth of the SiPM. Unfortunately, the bandwidth of the $\mathrm{J}$ series SiPMs is significantly higher than the $91 \mathrm{MHz}$ bandwidth of the commercial fluorescent fiber previously used in a VLC receiver [9]. Consequently, using this commercial fluorescent material with a SiPM would reduce the bandwidth of the receiver to less than $82 \mathrm{MHz}$. This negative impact on the receiver would be avoided using a fluorophore with a lifetime that is no larger than $0.64 \mathrm{~ns}$. In addition, to ensure that the photon detection efficiency of the SiPM is at least $80 \%$ of its peak value, the fluorophore should ideally predominantly emit light between $375 \mathrm{~nm}$ and $490 \mathrm{~nm}$.

One material which satisfies these requirements is pentafluorene (abcr $\mathrm{GmbH})$ in a poly(methyl methacrylate)

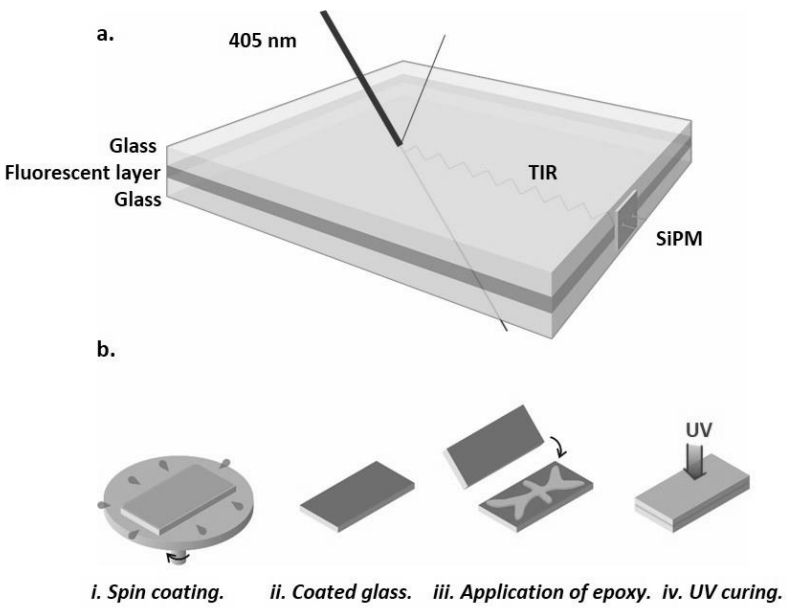

Fig 3. a) Concept of fluorescent antennas for VLC. A photon of incident light absorbed by the fluorophore incorporated within the fluorescent layer can result in the emission of a photon with a longer wavelength. Most of these emitted photons will be trapped within the antenna by total internal reflection (TIR) at the interface between glass and the air. Some of this trapped light eventually escapes the antenna from its edges, whose relatively small area can lead to an enhanced photon flux compared with that emitted from the top or bottom faces. The SiPM is therefore placed very close to one edge of the antenna. b) Fabrication process: i. The solution is spin-coated on a microscope slide. ii. A second microscope slide is spin-coated as well, and an optical epoxy is applied between them. iii. The glass/film/epoxy/film/glass structure is exposed to UV light for cure of epoxy.

(PMMA; Sigma-Aldrich) matrix. The photophysics of films of pentafluorene $(5 \mathrm{~F})$ in PMMA and the structure of $5 \mathrm{~F}$ are summarized in Fig. 2. These films show a strong absorption in the UVA region of the spectrum (peak absorption at $377 \mathrm{~nm}$, FWHM 351-401 nm) and emission in the deep blue region (vibronic 0-0 at $420 \mathrm{~nm}, 0-1$ at $441 \mathrm{~nm}, 0-2$ at $475 \mathrm{~nm}$, FWHM 410-456 nm). In addition, the measured PLQY was $90 \%$.

The fluorescence lifetime of 5F in a PMMA host was measured following short-pulse excitation with a diode laser and the results are shown in Fig. 2b. During a window of the first $6 \mathrm{~ns}$ following excitation, the fluorescence shows a single exponential decay with lifetime of $0.62 \pm 0.02$ ns. Since OOK signals are decoded at the center of each bit this lifetime, which corresponds to a $3 \mathrm{~dB}$ bandwidth of $257 \mathrm{MHz}$, is relevant for all data rates higher than $85 \mathrm{Mbps}$.

Fluorescent antennas that incorporate $5 \mathrm{~F}$ were next created using the steps illustrated in Fig. 3. Initially, a solution of $5 \mathrm{~F}$ and PMMA in $4 \mathrm{ml}$ of toluene (Sigma-Aldrich) was made, with $60 \mathrm{mg}$ of pentafluorene and $120 \mathrm{mg}$ of PMMA. Films were spin-coated at $1000 \mathrm{rpm}$ onto glass microscope slides (size: $75 \mathrm{~mm}$ x $25 \mathrm{~mm}$ x $1.1 \mathrm{~mm}$; Thermo Scientific). Two coated microscope slides were subsequently glued together using a UV-cured optical epoxy (NOA68; Norland). Spacers were used so that the final thickness of the epoxy was $0.1 \mathrm{~mm}$.

\section{CHARACTERIZATION OF THE FLUORESCENT ANTENNA}

The 5F antenna was characterized using a laser diode (Thorlabs L405P150) which emits $405 \mathrm{~nm}$. To ensure that the bandwidth measurements are relevant to the VLC link a network analyzer (HP-8712) was connected to the transmitter and receiver sides 


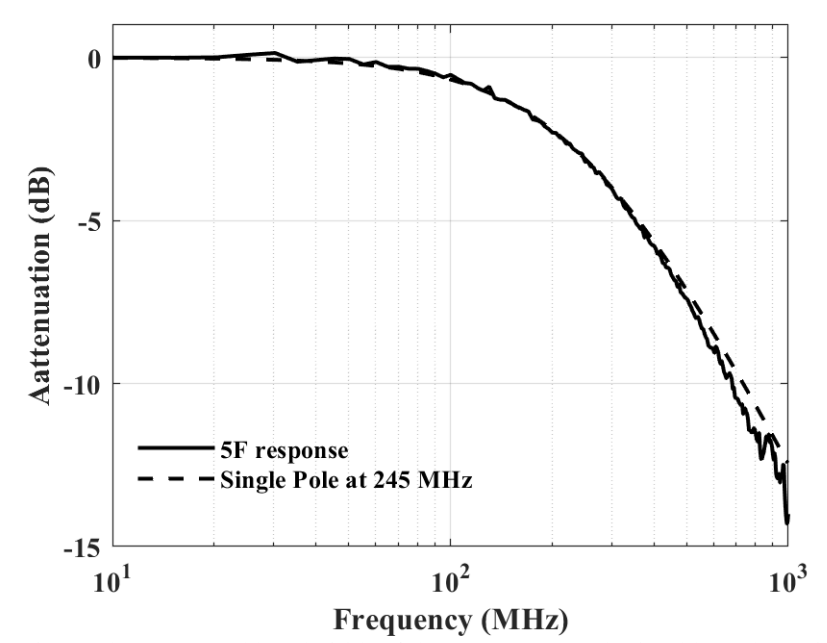

Fig. 4 The measured bandwidth of the $5 \mathrm{~F}$ antenna (obtained by subtracting the system response represented in $\mathrm{dB}$ when the receiver is the SiPM alone from the equivalent response when the receiver is the $5 \mathrm{~F}$ sample and the

SiPM) compared to a single pole response with a $3 \mathrm{~dB}$ bandwidth of $245 \mathrm{MHz}$.

of the data transmission experimental setup described in section $\mathrm{V}$. At frequencies less than $30 \mathrm{MHz}$, the systems response is influenced by the $10 \mathrm{MHz}-6 \mathrm{GHz} \mathrm{RF}$ amplifier (FMAM3269) required to drive the laser diode. The frequency response is then frequency independent between $30 \mathrm{MHz}$ and $100 \mathrm{MHz}$. Using this region as the $0 \mathrm{~dB}$ level the measured 3 $\mathrm{dB}$ frequency of the SiPM was $257 \mathrm{MHz}$. This is close to the $249 \mathrm{MHz}$ estimated from the FWHM of the fast output pulses in the data sheet.

The frequency response of the $5 \mathrm{~F}$ antenna was obtained from the changes in the frequency response of the system when the $5 \mathrm{~F}$ antenna was included in the receiver, and is shown in Fig. 4. The results show that the frequency response of the $5 \mathrm{~F}$ antenna is very similar to that of a system with a single exponential response. Furthermore, the bandwidth of the antenna is $245 \mathrm{MHz}$. The method used to construct the antenna and its geometry have therefore not significantly reduced its bandwidth compared to the 5F in PMMA film, which has a bandwidth of $257 \mathrm{MHz}$. Consequently, the bandwidth of this antenna is 2.7 times the bandwidth of the commercial material used previously in an antenna[9].

Placing our antenna between the laser diode and an optical power meter covered by a $405 \mathrm{~nm}$ filter showed that the antenna absorbs $32 \%$ of the incident $405 \mathrm{~nm}$ light. A Mylar mirror was placed behind the antenna reflects any $405 \mathrm{~nm}$ light that passes through the antenna back through it, leading to overall absorption of approximately $50 \%$ of the incident $405 \mathrm{~nm}$ light.

The impact of 500 lux of ambient light from Philips $(5.5 \mathrm{~W}+8 \mathrm{~W})$ A60 2700K warm white LEDs on the SiPM was determined by exposing the SiPM to this light and measuring the current required to maintain the SiPM bias voltage. A comparison of this SiPM bias current with the results in Fig 1 showed that this ambient light was equivalent to an irradiance of $200 \mathrm{mWm}^{-2}$ of $405 \mathrm{~nm}$ light. When the SiPM was coupled to the edge of the $5 \mathrm{~F}$ antenna the bias current reduced to the equivalent of an irradiance of $10 \mathrm{mWm}^{-2}$.

The selective absorption of the 5F should ensure that most of the ambient light passes through the antenna and so the

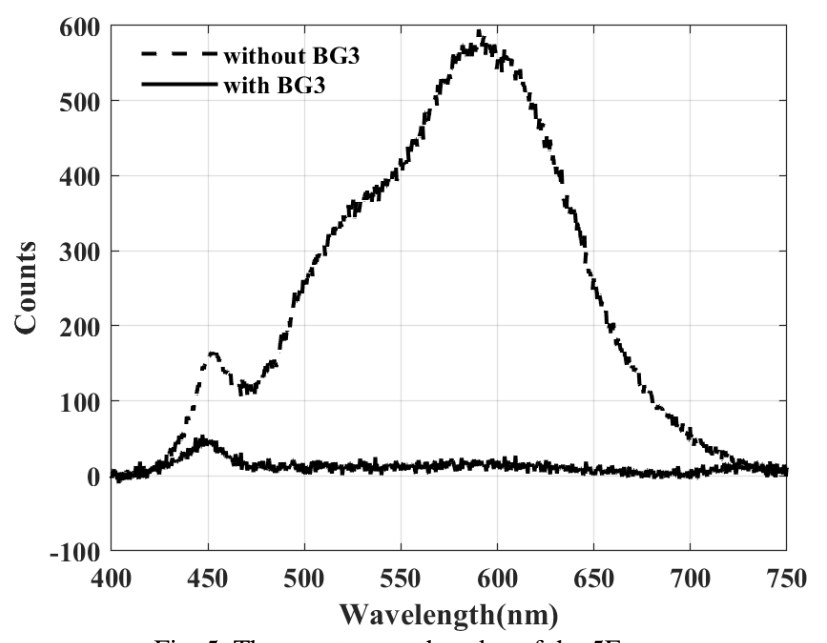

Fig. 5 The spectrum at the edge of the $5 \mathrm{~F}$ antenna

observed reduction in the SiPM bias current was smaller than expected. The spectrum of light at the edge of the antenna when it is exposed to ambient light was therefore measured. Figure 5 shows that unexpectedly ambient light is present at the edge of the antenna. Since the spectrometer input fiber was shielded from ambient light the observed ambient light must arise from extrinsic scattering within the antenna.

The amount of ambient light reaching the edges of the antenna can be reduced further by placing an optical filter in front of the $5 \mathrm{~F}$ antenna. To preserve the wide field of view of the receiver an absorption filter (3 mm thick Schott glass BG3) was used. As shown in Fig. 5 this significantly reduces the amount of ambient light reaching the edge of the $5 \mathrm{~F}$ antenna. Overall, the combination of the BG3 filter and the 5F antenna reduces the effect of ambient light on the SiPM by a factor of 200.

In addition to filtering a possible advantage of a fluorescent optical element is that it can increase the irradiance falling in the photodetector. The exposed area of the 5F antenna is 111 times the area of the SiPM. However, the SiPM only detects light from a $3 \mathrm{~mm}$ section of the $150 \mathrm{~mm}$ perimeter of the antenna. If the irradiance is uniform around the perimeter the maximum gain of the antenna will be approximately 2.2. However, only $50 \%$ of the incident light is absorbed by the $5 \mathrm{~F}$ antenna and approximately $75 \%$ of the light is then retained within the antenna. Consequently, the maximum estimated increase in irradiance falling on the SiPM when the antenna is used is 0.83 .

To compare the received transmitter signals with and without the 5F antenna, the SiPM bias currents have been measured for the same constant irradiance from the $405 \mathrm{~nm}$ transmitter. When the SiPM was irradiated directly the SiPM bias current was $4.96 \mathrm{~mA}$. However, when the SiPM was coupled to the $5 \mathrm{~F}$ antenna and exposed to the same irradiance the SiPM bias current fell to $1.39 \mathrm{~mA}$. Since these currents are proportional to the rate at which photons are detected it appears that scattering in the antenna is reducing the amount of light retained within it. Consequently, the SiPM signal is even lower than predicted. In fact when used with this SiPM the 5F antenna reduces the signal from the transmitter at the SiPM output by a factor of 0.28 . 


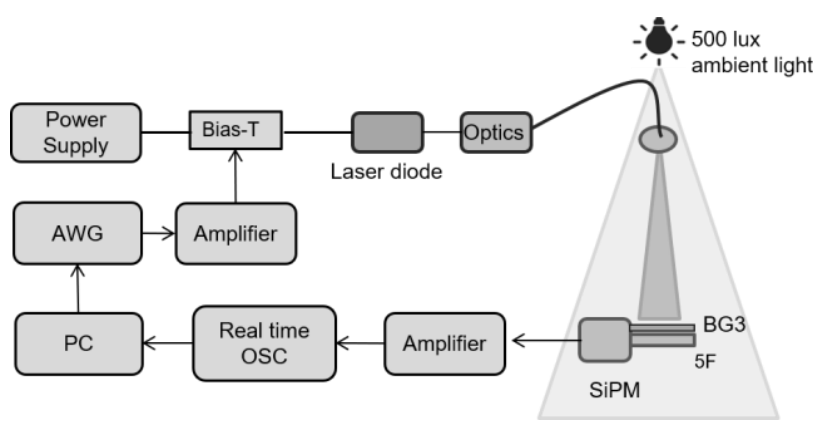

Fig. 6 A schematic diagram of the experiments used to determine the irradiance required to support different OOK data rates at a BER of $10^{-3}$. The distance between the transmitter and the receiver was $30 \mathrm{~cm}$.

Hence in our experiments, the antenna is not an optical concentrator.

\section{DATA TRANSMISSION EXPERIMENTS}

A schematic diagram of the experimental setup used for determining the performance of VLC receivers is shown in Fig 6. This setup consisted of a $10 \mathrm{GHz}$ Tektronix arbitrary waveform generator (AWG) which generated an OOK pseudorandom binary sequence (PRBS) signal. A Fairview microwave (FMAM3269) amplifier was connected to this AWG to amplify its maximum output signal from $500 \mathrm{mVpp}$ to 2 Vpp. However, because this amplifier is not effective at frequencies below $10 \mathrm{MHz}, 8 \mathrm{~b} 10 \mathrm{~b}$ coding was employed. After amplification the output signal was added to a DC bias using a Bias-Tee (ZFBT-4R2GW+). The signal from the Bias-Tee was applied to Thorlabs L405P150 laser diode (with peak wavelength of $405 \mathrm{~nm}$ ). A polarizer in front of the laser diode was used to vary the irradiance from this laser diode falling on the SiPM. The output light from the polarizer was then coupled into a multimode fibre by a fibre collimator. A diffuser and plano-convex lens were used at the other end of the fibre to create a diffuse beam. To provide representative operating conditions the receiver was also illuminated by 500 lux of ambient light from the Philips $(5.5 \mathrm{~W}+8 \mathrm{~W})$ A60 2700K warm white LEDs.

The photodetector in the receiver was a J-series 30020 SiPM, whose key performance parameters are listed in Table 1. The fast output of the SiPM was amplified using a ZFL-1000LN+ amplifier. The fast output pulse of the J Series SiPMs have a sharp rising edge followed by an exponential decay. The full width half maximum (FWHM) of the fast output pulses from the $30020 \mathrm{SiPM}$ used in the experiments described in the paper is $1.4 \mathrm{~ns}$ (Table 1 ). The target BER, $10^{-3}$, is only achieved when at least 6 photons are detected during any time interval in which a ' 1 ' is being transmitted. For the experiments reported in the paper, the longest time interval used to transmit a bit was $5 \mathrm{~ns}$ (200 Mbps). Consequently, even at the lowest reported data rate, the average time between detected photons is smaller than the width of the fast output pulses. The resulting overlap between the pulses generated by the individual photons means that the voltage on the SiPMs fast output was an analogue voltage, which was captured by a Tektronix MSO64 (4 GHz, $24 \mathrm{GS} / \mathrm{s}$ ) oscilloscope (OSC). Finally, the captured data was processed offline using MATLAB $^{\circledR}$. This post processing

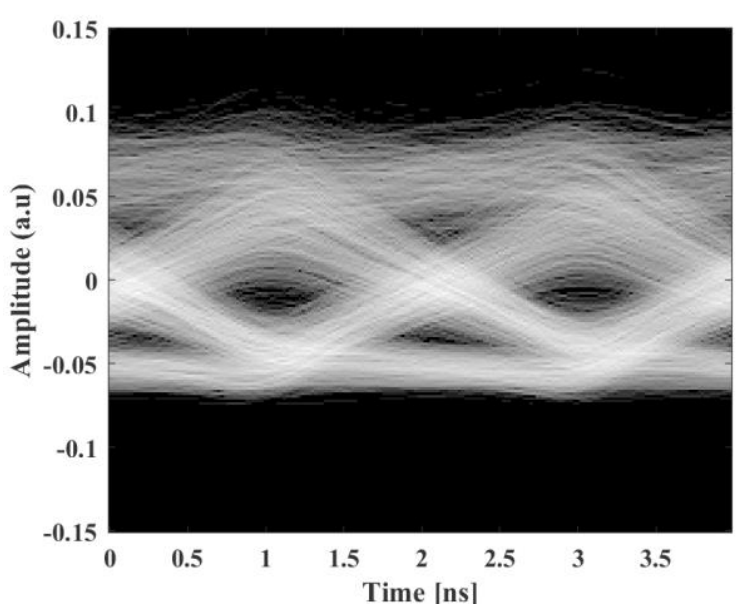

Fig. 7 The eye diagram at $500 \mathrm{Mbps}$ in 500 lux of ambient light measured with a receiver consisting of a BG3 filter, $5 \mathrm{~F}$ antenna and SiPM

included applying decision feedback equalization (DFE) before determining the resulting bit error rate (BER). In particular, the BER results reported in this paper were calculated by transmitting 10 different sequences of $2^{15}$ pseudo-random bits. For each OOK data rate the irradiance was varied until the BER was $10^{-3}$. The average received irradiance from the transmitter for each data rate was then measured using the Newport 818SL power meter.

Using this approach to measuring the performance of the receiver experiments were performed with the SiPM covered by a BG3 filter. In addition, experiments were performed with the SiPM enclosed in a box and coupled to part of one short edge of the 5F antenna inserted into a slot in the box. Finally, experiments were performed with a $50.8 \mathrm{~mm}$ by $50.8 \mathrm{~mm}$ BG3 filter on top of $40 \mathrm{~mm}$ by $25 \mathrm{~mm}$ exposed area of the $5 \mathrm{~F}$ antenna. A representative eye diagram from this final set of experiments, when the BG3 filter and 5F antenna are used, with the SiPM, at a data rate of $500 \mathrm{Mbps}$ is shown in Fig. 7.

The results of experiments with the three different receiver configurations are shown in Fig. 8. Adding a BG3 filter to a receiver formed by the $5 \mathrm{~F}$ antenna and the $\mathrm{SiPM}$ reduces the

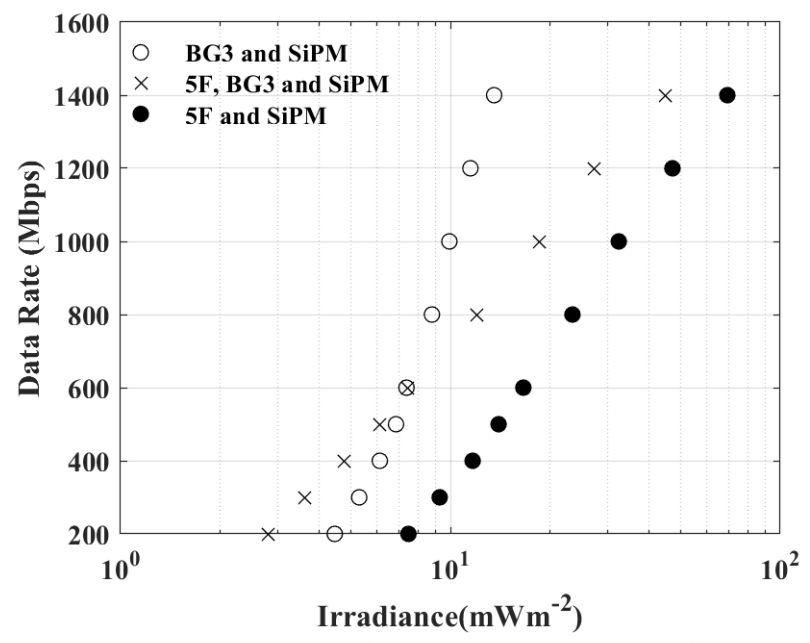

Fig. 8 A comparison of the irradiances required to support different data rates for three different receivers when ambient light at 500 lux is present. 


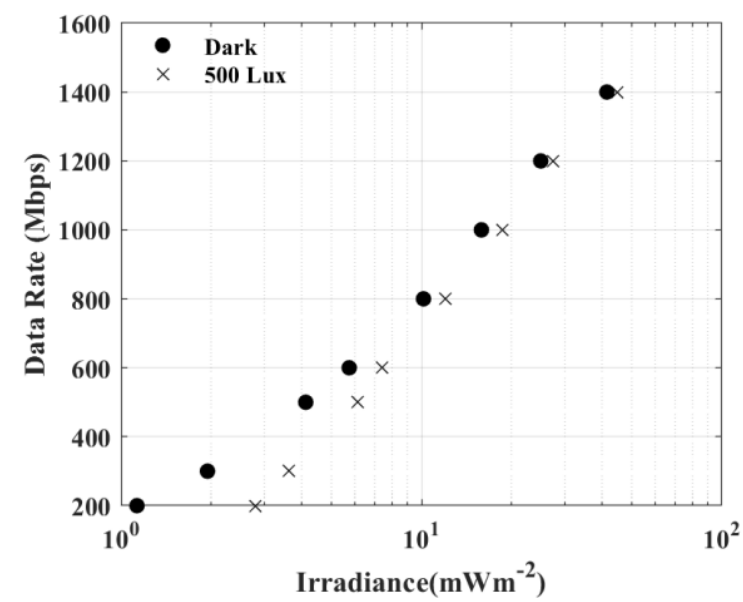

Fig. 9 A comparison of the irradiances required to support different data rates in the dark and in 500 lux of ambient light for a receiver consisting of a BG3 filter, 5F antenna and SiPM.

rate at which ambient light photons are detected by the SiPM by a factor of 10. If the dominant noise source in the SiPM is Poisson or shot noise from the ambient light then adding the BG3 filter to the receiver will reduce the receiver's noise by a factor of 3.3. Consequently, adding the BG3 filter would be expected to reduce the irradiance required to support a required BER by a factor of 3.3. The results in Fig. 8 show that adding the BG3 to the receiver reduced the irradiance required to support each data rate. At $200 \mathrm{Mbps}$ adding the BG3 filter reduces the required irradiance by a factor of 2.7. At this data rate the Poisson or shot noise from the ambient light is therefore the most significant source of noise. However, the increased irradiance needed to support higher data rates means that Poisson/shot noise from the transmitted signal becomes more significant. Despite this trend, adding the BG3 filter reduces the irradiance required to transmit $1.4 \mathrm{Gbps}$ by a factor of 1.4 . Equivalently, at $47 \mathrm{mWm}^{-2}$ adding the BG3 filter increases the data rate from $1.2 \mathrm{Gbps}$ to $1.4 \mathrm{Gbps}$ whilst at an irradiance of $7.4 \mathrm{mWm}^{-2}$ the data rate increases from $200 \mathrm{Mbps}$ to $600 \mathrm{Mbps}$.

The results in Fig. 8 also show that the impact of adding the $5 \mathrm{~F}$ antenna to the receiver formed by the SiPM and BG3 filter depends upon data rate. At data rates more than $600 \mathrm{Mbps}$ the lifetime of the fluorophore has a negative impact on the receiver's performance. Consequently, adding the $5 \mathrm{~F}$ antenna to the receiver increases the irradiance required to support each data rate. However, at lower data rates the $5 \mathrm{~F}$ antenna reduces the irradiance required to support a particular OOK data rate. For example at $200 \mathrm{Mbps}$, adding the $5 \mathrm{~F}$ antenna to the receiver reduces the required irradiance by a factor of 1.6. Alternatively, at lower data rates adding the $5 \mathrm{~F}$ antenna approximately doubles the achievable data rate at the same irradiance, from $200 \mathrm{Mbps}$ to approximately $400 \mathrm{Mbps}$. These improvements in performance occur despite the fact that the introduction of the $5 \mathrm{~F}$ antenna reduces the transmitted irradiance falling on the SiPM. The improvements arising from including the $5 \mathrm{~F}$ antenna in the receiver are therefore entirely due to it acting as a filter.

Fig. 9 shows the data rates achievable in the dark and in 500 lux of ambient light. These results show that when the BG3 filter and the $5 \mathrm{~F}$ antenna are used with the SiPM operation at $200 \mathrm{Mbps}$ in 500 lux of ambient light requires an increase in irradiance of a factor of only $2.5(4 \mathrm{~dB})$. Furthermore, as the irradiance from the transmitter increases to support higher data rates the increase in irradiance required to operate in ambient light reduces. Consequently, the increase in irradiance required to support $1 \mathrm{Gbps}$ in ambient light is only $1.2(0.8 \mathrm{~dB})$ times higher than for transmission in the dark. The BG3 filter and 5F antenna therefore largely protect the SiPM from ambient light. Consequently, the receiver's performance in ambient light is very close its performance in the dark.

\section{CONCLUSION}

In this paper the results from a receiver formed from a novel combination of a fluorescent antenna and a SiPM are reported for the first time. The fluorescent antenna contained pentafluorene, which has a fluorescence lifetime of $0.62 \mathrm{~ns}$. Consequently, the bandwidth of a rectangular fluorescent antenna containing pentafluorene was shown to be $245 \mathrm{MHz}$, which is 2.7 times the bandwidth of a commercial fluorophore doped optical fiber used previously[9].

The results of data transmission experiments in 500 lux of ambient light show that the antenna's selective response to the light from the transmitter reduced the irradiance needed to transmit a particular data rate and doubles the achievable data rate at the same irradiance. Furthermore, when the BG3 filter and the $5 \mathrm{~F}$ antenna are both used, $1 \mathrm{Gbps}$ data transmission in 500 lux of ambient light needed only $1.2(0.8 \mathrm{~dB})$ times higher irradiance than in the absence of ambient light.

Overall, these results show that wide field of view receivers incorporating extremely sensitive SiPMs can operate in the presence of ambient light in 'real world' environments. Further work is required to improve the bandwidth and gain of available antennas. One promising approach to improving the bandwidth of wide field of view VLC antennas, which has recently been reported, is to combine fluorophores with nanopatch antennas [12].

\section{ACKNOWLEDGMENT}

We are grateful to Dr Kou Yoshida for assistance with the PLQY measurements and to Dr Grahame Faulkner for assistance with the communications measurements.

\section{REFERENCES}

[1] J. Lian, Z. Vatansever, M. Noshad, and M. Brandt-Pearce, "Indoor visible light communications, networking, and applications," JPhys Photonics, vol. 1, no. 1, 2019, doi: 10.1088/2515-7647/aaf74a.

[2] A. Jovicic, J. Li, and T. Richardson, "Visible light communication:Opportunities, challenges and the path to market,"IEEE Commun. Mag.,vol. 51, no. 12, pp. 26-32, Dec. 2013.W.-K. Chen, Linear Networks and Systems. Belmont, CA, USA: Wadsworth, 1993, pp. 123135.

[3] R. Bian, I. Tavakkolnia, and H. Haas, "15.73 Gb/s Visible Light Communication with Off-the-Shelf LEDs," J. Light. Technol., vol. 37, no. 10, pp. 2418-2424, 2019, doi: 10.1109/JLT.2019.2906464.

[4] H. Chun, A. Gomez, C. Quintana, W. Zhang, G. Faulkner, and D. O'Brien, "A Wide-Area Coverage $35 \mathrm{~Gb} / \mathrm{s}$ Visible Light Communications Link for Indoor Wireless Applications," Sci. Rep., vol. 9, no. 1, pp. 4-11, 2019, doi: 10.1038/s41598-019-41397-6.

[5] L.-Y. Wei, C.-W. Chow, G.-H. Chen, Y. Liu, C.-H. Yeh, and C.-W. Hsu, "Tricolor visible-light laser diodes based visible light communication operated at $40.665 \mathrm{Gbit} / \mathrm{s}$ and $2 \mathrm{~m}$ free-space transmission," Opt. Express, vol. 27 , no. 18, p. 25072, 2019, doi: 10.1364/oe.27.025072.

[6] W. Matthews, W. Ali, Z. Ahmed, G. Faulkner, and S. Collins, "Intersymbol interference and silicon photomultiplier VLC receivers in ambient 
light," IEEE Photonics Technol. Lett., vol. 33, no. 9, pp. 449-452, 2021, doi: 10.1109/LPT.2021.3067511.

[7] Z. Ahmed, R. Singh, W. Ali, G. Faulkner, D. O'Brien and S. Collins, "A SiPM-Based VLC Receiver for Gigabit Communication Using OOK Modulation," in IEEE Photonics Technology Letters, vol. 32, no. 6, pp. 317-320, March 2020.

[8] Pavlos P. Manousiadis, Sujan Rajbhandari, Rahmat Mulyawan, Dimali A. Vithanage, Hyunchae Chun, Grahame Faulkner, Dominic C. O'Brien, Graham A. Turnbull, Stephen Collins, and Ifor D.W. Samuel, "Wide fieldof-view fluorescent antenna for visible light communications beyond the étendue limit," Optica 3, 702-706 (2016)

[9] T. Peyronel, K. J. Quirk, S. C. Wang, and T. G. Tiecke, "Luminescent detector for free-space optical communication," Optica 3, 787-792 (2016)

[10] Steve Collins, Dominic C. O'Brien, and Andrew Watt, "High gain, wide field of view concentrator for optical communications," Opt. Lett. 39, 1756-1759 (2014)

[11] Onsemi.com. 2020. J-Series SiPM Sensors Datasheet. [online] Available at: https://www.onsemi.com/pub/Collateral/MICROJ-SERIES-D.PDF, Accessed 10 March 2020

[12]Rasskazov, Ilia L., Alexander Moroz, and P. Scott Carney. "Extraordinary fluorescence enhancement in metal-dielectric core-shell nanoparticles." arXiv preprint arXiv:2104.00056 (2021). 